\title{
Dilute magnetic semiconducting behaviour of Fe doped CdSe nanoparticles
}

\author{
T. P. Sinha ${ }^{1 *}$, Sayantani Das ${ }^{2}$, S. Banerjee ${ }^{2}$ and S. Bandopadhay ${ }^{2}$
}

${ }^{1}$ Department of Physics, Bose Institute, 93/1, Acharya Prafulla Chandra Road, Kolkata 700009, India

${ }^{2}$ Department of Physics, University of Calcutta, 92, Acharya Prafulla Chandra Road, Kolkata-700009, India

*Email: sinha_tp@yahoo.com

Cadmium Selenide (CdSe) has become an interesting member of the II-VI semiconductor family due to its high photosensitivity and suitable band gap (1.74 eV at $300 \mathrm{~K})$ [1]. Iron is a prominent dopant in II-VI semiconductors and is thus very suitable as a model system to study the influence of the host crystal to an impurity ion [2]. In the present work we have prepared pure and $\mathrm{Fe}(5 \%$ and $10 \%)$ doped $\mathrm{CdSe}$ nanoparticles (hence forth referred as $\mathrm{CdFeSe} 1$ and $\mathrm{CdFeSe} 2$ respectively) using the soft chemical technique which is an inexpensive route with less instrumentation and operated at room temperature. The $\mathrm{x}$-ray diffraction (XRD) pattern indicates the cubic structure of the synthesized materials. The absence of any peak for Fe ions (in the XRD patterns of $\mathrm{CdFeSe} 1$ and $\mathrm{CdFeSe} 2$ ) indicates the complete substitution of $\mathrm{Fe}$ ions in $\mathrm{CdSe}$ lattice sites without any secondary phase or precipitate in the samples. We have carried out the transmission electron microscope (TEM) of $\mathrm{CdSe}, \mathrm{CdFeSe} 1$ and $\mathrm{CdFeSe} 2$ respectively to find out the shape and size of the synthesized nanoparticles. SAED pattern supports the cubic structure of the materials.

Raman spectrum of the samples is taken in the range of $80-500 \mathrm{~cm}^{-1}$. The Raman spectra are analyzed to obtain the $1^{\text {st }}$ and $2^{\text {nd }}$ order phonon modes (longitudinal and transverse optical modes) of the samples. The spectra exhibit a number of deconvoluted peaks along with the main peaks. A sum of 9 Lorentzian peaks is used to fit each of the Raman spectra.

The field cooled (FC) and zero field cooled (ZFC) magnetization (M) of the samples are measured as a function of temperature. Figure 1 shows the magnetization vs. magnetic field $(\mathrm{M}-\mathrm{H})$ curve at $5 \mathrm{~K}$ for $\mathrm{CdFeSe} 2$. From this figure we can say that the sample is antiferromagnetic in nature showing the value of $\mathrm{M}=51 \mathrm{emu} / \mathrm{gm}$ for $\mathrm{H}=10 \mathrm{kOe}$. We have extracted the ferromagnetic contribution of the sample from this curve (as shown in the inset of Figure 1) for which $M=12 \mathrm{emu} / \mathrm{gm}$. So the ferromagnetic contribution of the sample at $5 \mathrm{~K}$ is about $23 \%$. We have also calculated the same for CdFeSe1.

The temperature $(\mathrm{T})$ dependence of the inverse molar magnetic susceptibility $\left(1 / \chi_{\mathrm{m}}\right)$ of $\mathrm{CdFeSe} 1$ and $\mathrm{CdFeSe} 2$ at the field of $500 \mathrm{Oe}$ is plotted to find out the effective Bohr magneton of the sample. A linear fit to the experimental data at higher temperatures by Curie-Weiss law yields a Curie-Weiss constant $(\Theta)$ for $\mathrm{CdFeSe} 1$ and $\mathrm{CdFeSe} 2$ of $50.79 \mathrm{~K}$ and $242.5 \mathrm{~K}$ respectively. The effective Bohr magneton number $\left(\mathrm{P}_{\text {eff }}\right)$ of $\mathrm{CdFeSe} 1$ and $\mathrm{CdFeSe} 2$ nanomaterials is found to be $1.389 \mu_{\mathrm{B}}$ and $1.9007 \mu_{\mathrm{B}}$ per formula unit respectively using the following equation $\mathrm{P}_{\mathrm{eff}}=2.827(\mathrm{C})^{1 / 2}$, where $\mathrm{C}$ is the Curie constant.

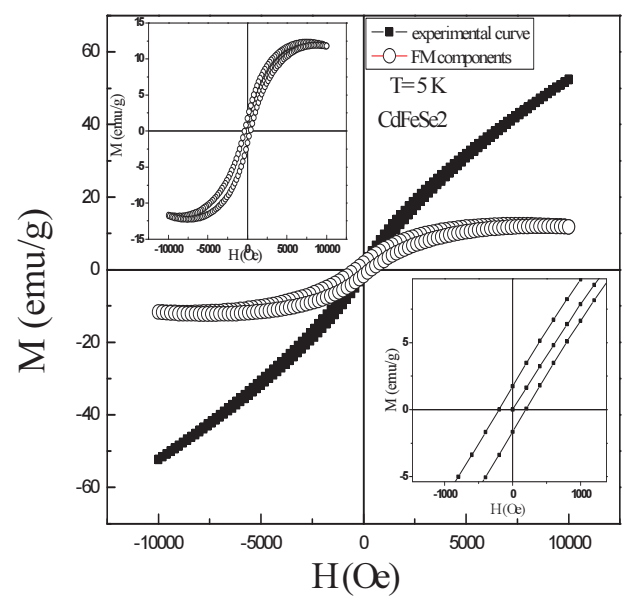

Figure 1: M-H curve of CdFeSe2

\section{References}

1. S. Das, A. Dutta, B. Ghosh, S. Banerjee and T. P. Sinha, J. Phys. Chem. Sol. 75 (2014) 1245.

2. S. Das, A. Dutta, S. Banerjee and T. P. Sinha, Mat. Sc. Semicond. Process.18 (2014) 152. 